\title{
High mobility and high thermoelectric power factor in epitaxial ScN thin films deposited with plasma-assisted molecular beam epitaxy ${ }^{\circledR}$
}

Cite as: Appl. Phys. Lett. 116, 152103 (2020); https://doi.org/10.1063/5.0004761

Submitted: 14 February 2020 . Accepted: 25 March 2020 . Published Online: 14 April 2020

(D) Dheemahi Rao, (D) Bidesh Biswas, Eduardo Flores, Abhijit Chatterjee, (iD) Magnus Garbrecht, (D) Yee Rui Koh, Vijay Bhatia, Ashalatha Indiradevi Kamalasanan Pillai, (D) Patrick E. Hopkins, (D) Marisol Martin-Gonzalez, and (i) Bivas Saha

COLLECTIONS

EP This paper was selected as an Editor's Pick
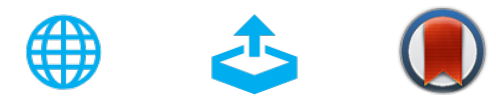

\section{ARTICLES YOU MAY BE INTERESTED IN}

Molecular beam epitaxy and characterization of wurtzite $\mathrm{Sc}_{x} \mathrm{Al}_{1-x} \mathrm{~N}$

Applied Physics Letters 116, 151903 (2020); https://doi.org/10.1063/5.0002445

Demonstration and aging test of a radiation resistant strontium-90 betavoltaic mechanism Applied Physics Letters 116, 153901 (2020); https://doi.org/10.1063/1.5140780

Field-programmable acoustic array for patterning micro-objects

Applied Physics Letters 116, 151901 (2020); https://doi.org/10.1063/5.0003147

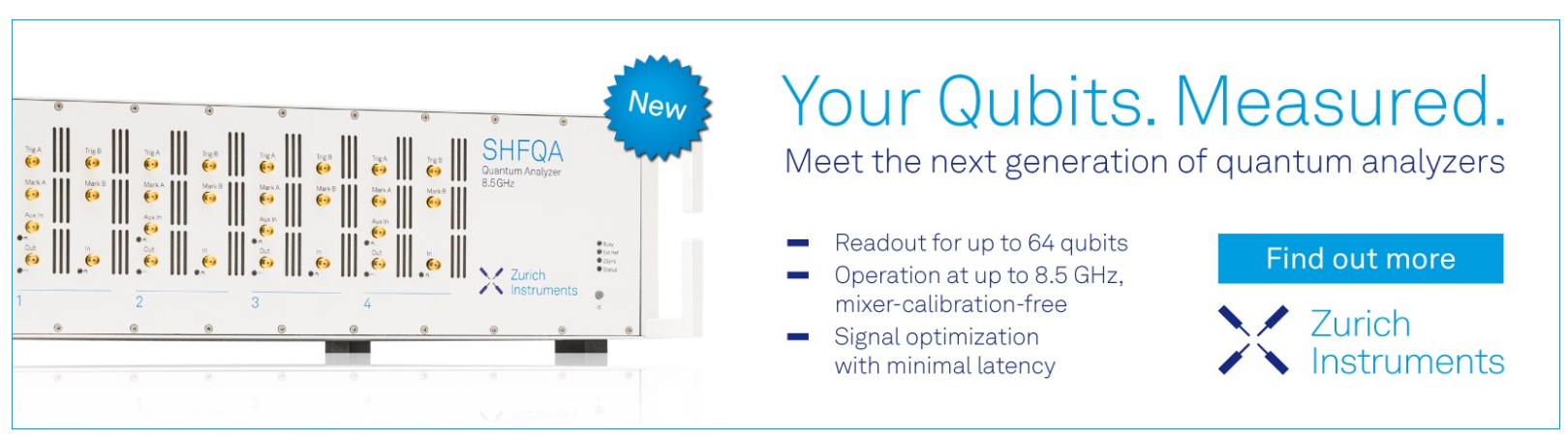




\title{
High mobility and high thermoelectric power factor in epitaxial ScN thin films deposited with plasma-assisted molecular beam epitaxy 루
}

Cite as: Appl. Phys. Lett. 116, 152103 (2020); doi: 10.1063/5.0004761

Submitted: 14 February 2020 - Accepted: 25 March 2020 .

Published Online: 14 April 2020

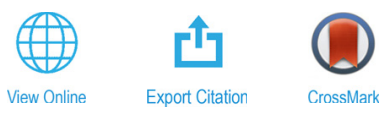

Dheemahi Rao, ${ }^{1,2,3}$ (D) Bidesh Biswas, ${ }^{1,2,3}$ (D) Eduardo Flores, ${ }^{4}$ Abhijit Chatterjee, ${ }^{1,2,3}$ Magnus Garbrecht, ${ }^{5}$ Yee Rui Koh, ${ }^{6}$ iD Vijay Bhatia, ${ }^{5}$ Ashalatha Indiradevi Kamalasanan Pillai, ${ }^{5}$ Patrick E. Hopkins, ${ }^{6,7,8}$ (iD) Marisol Martin-Gonzalez, ${ }^{4}$ (iD and Bivas Saha, ${ }^{1,2,3, a)}$ (iD)

\begin{abstract}
AFFILIATIONS
${ }^{1}$ Chemistry and Physics of Materials Unit, Jawaharlal Nehru Centre for Advanced Scientific Research, Bangalore 560064, India ${ }^{2}$ International Centre for Materials Science, Jawaharlal Nehru Centre for Advanced Scientific Research, Bangalore 560064, India

${ }^{3}$ School of Advanced Materials, Jawaharlal Nehru Centre for Advanced Scientific Research, Bangalore 560064, India

${ }^{4}$ Instituto de Micro and Nanotecnologia, IMN-CSIC, C/Isaac Newton 8, Tres Cantos, 28760 Madrid, Spain

${ }^{5}$ Australian Centre for Microscopy and Microanalysis, The University of Sydney, Camperdown, NSW 2006, Australia

${ }^{6}$ Department of Mechanical and Aerospace Engineering, University of Virginia, Charlottesville, Virginia 22904, USA

${ }^{7}$ Department of Materials Science and Engineering, University of Virginia, Charlottesville, Virginia 22904, USA

${ }^{8}$ Department of Physics, University of Virginia, Charlottesville, Virginia 22904, USA
\end{abstract}

a) Author to whom correspondence should be addressed: bsaha@jncasr.ac.in and bivas.mat@gmail.com

\begin{abstract}
Scandium nitride $(\mathrm{ScN})$ is an emerging rock salt III-nitride semiconductor and has attracted significant interest in recent years for its potential thermoelectric applications as a substrate for high-quality epitaxial GaN growth and as a semiconducting component for epitaxial singlecrystalline metal/semiconductor superlattices for thermionic energy conversion. Solid-solution alloys of ScN with traditional III-nitrides such as $\mathrm{Al}_{\mathrm{x}} \mathrm{Sc}_{1-\mathrm{x}} \mathrm{N}$ have demonstrated piezoelectric and ferroelectric properties and are actively researched for device applications. While most of these exciting developments in $\mathrm{ScN}$ research have employed films deposited using low-vacuum methods such as magnetron sputtering and physical and chemical vapor depositions for thermoelectric applications and Schottky barrier-based thermionic energy conversion, it is necessary and important to avoid impurities, tune the carrier concentrations, and achieve high-mobility in epitaxial films. Here, we report the high-mobility and high-thermoelectric power factor in epitaxial $\mathrm{ScN}$ thin films deposited on $\mathrm{MgO}$ substrates by plasma-assisted molecular beam epitaxy. Microstructural characterization shows epitaxial 002 oriented $\mathrm{ScN}$ film growth on $\mathrm{MgO}$ (001) substrates. Electrical measurements demonstrated a high room-temperature mobility of $127 \mathrm{~cm}^{2} / \mathrm{V} \mathrm{s}$ and temperature-dependent mobility in the temperature range of $50-400 \mathrm{~K}$ that is dominated by dislocation and grain boundary scattering. High mobility in $\mathrm{ScN}$ films leads to large Seebeck coefficients $(-175 \mu \mathrm{V} / \mathrm{K}$ at $950 \mathrm{~K})$ and, along with a moderately high electrical conductivity, a large thermoelectric power factor $\left(2.3 \times 10^{-3} \mathrm{~W} / \mathrm{m}-\mathrm{K}^{2}\right.$ at $500 \mathrm{~K}$ ) was achieved, which makes $\mathrm{ScN}$ a promising candidate for thermoelectric applications. The thermal conductivity of the films, however, was found to be a bit large, which resulted in a maximum figure-of-merit of 0.17 at $500 \mathrm{~K}$.
\end{abstract}

Published under license by AIP Publishing. https://doi.org/10.1063/5.0004761

Transition metal nitrides (TMNs) are an interesting class of materials and have attracted significant interest in recent years for a range of energy conversion applications such as solarthermophotovoltaics (STPVs), ${ }^{1}$ photo-catalysis, ${ }^{2}$ thermoelectrics, ${ }^{3-}$ and thermionic energy conversion. ${ }^{6,7}$ Scandium nitride $(\mathrm{ScN})$ is the most famous semiconducting TMN and exhibits corrosion-resistant high mechanical hardness $(\sim 21 \mathrm{GPa}),{ }^{8}$ structural, morphologic, and thermodynamic stabilities, ${ }^{9}$ and large melting temperatures in excess of $2600^{\circ} \mathrm{C} .{ }^{10,11}$ Unlike the other traditional wurtzite III-nitride semiconductors such as $\mathrm{GaN}$ and $\mathrm{InN}, \mathrm{ScN}$ crystallizes in the rock salt structure with octahedral bonding coordination ${ }^{12,13}$ and exhibits an indirect bandgap of $\sim 0.9 \mathrm{eV}$ and a direct gap of $2.2 \mathrm{eV} .{ }^{14-16}$ Due to its cubic (rock salt) crystal structure and low bandgap, $\mathrm{ScN}$ has been utilized in the development of the first epitaxial rock salt 
single-crystalline $\mathrm{ZrN} / \mathrm{ScN}^{17,18}$ and $\left.\mathrm{TiN} /(\mathrm{Al}, \mathrm{Sc}) \mathrm{N}\right)^{19-21}$ metal/semiconductor multilayers and superlattices, respectively, for Schottky diode-based thermionic energy conversion. $\mathrm{ScN}$ has also attracted significant interest from thermoelectric research communities as it exhibits a large thermoelectric power factor $\left(S^{2} \sigma\right)$ (S and $\sigma$ are the Seebeck coefficient and electrical conductivity, respectively) due to the high Seebeck coefficient and moderately high electrical conductivity resulting from its degenerate semiconducting nature. Sputter-deposited ScN thin films on $\mathrm{MgO}$ and sapphire substrates have shown a large thermoelectric power factor of $\sim 3.3 \times 10^{-3} \mathrm{~W} / \mathrm{m}-\mathrm{K}^{2}$ in the temperature range of $500-800 \mathrm{~K}$, which is higher than that of many well-known thermoelectric materials such as $\mathrm{Bi}_{2} \mathrm{Te}_{3}$ in the same temperature range. $^{4,22}$

In addition to their thermoelectric properties, solid-solution alloys of ScN with other III-nitride semiconductors such as $\mathrm{GaN}^{23}$ have attracted interest for band structure engineering of GaN-based light-emitting diodes (LEDs) with a reduced indium content and for achieving green LEDs with all nitride semiconductors. ${ }^{24}$ Moreover, since the lattice constant of $\operatorname{ScN}(a=4.50 \AA)^{16}$ and the in-plane lattice parameter of $\mathrm{GaN}(a=3.18 \AA)^{25}$ result in a small $0.1 \%$ mismatch for the (0001) oriented GaN growth on (111) ScN surfaces, $\mathrm{ScN}$ has been utilized as a substrate for reducing the threading dislocation densities in GaN epilayers. ${ }^{26,27}$ Wurtzite $\mathrm{Al}_{\mathrm{x}} \mathrm{Sc}_{1-\mathrm{x}} \mathrm{N}$ solid-solution alloys have exhibited a large $c$-axis piezoelectric coefficient that is higher than that of AlN, and it is currently researched for the development of bulk and surface acoustic devices. ${ }^{28}$ Recently, $\mathrm{Al}_{\mathrm{x}} \mathrm{Sc}_{1-\mathrm{x}} \mathrm{N}$ has also demonstrated ferroelectricity with a continuous distortion of the wurtzite structure to a layered hexagonal phase with the increasing scandium concentration and a ferroelectric-to-paraelectric transition temperature higher than $600{ }^{\circ} \mathrm{C} .{ }^{29}$ Wurtzite $\mathrm{Al}_{\mathrm{x}} \mathrm{Sc}_{1-\mathrm{x}} \mathrm{N}$ has also shown promise for highpower electronic and optoelectronic device applications. ${ }^{30}$

Sputter-deposited $\mathrm{ScN}$ possesses a high $n$-type carrier concentration of $(1-5) \times 10^{20} \mathrm{~cm}^{-3}$ due to the presence of unwanted impurities such as oxygen $\left(\mathrm{O}_{\mathrm{N}}\right)$ and fluorine $\left(\mathrm{F}_{\mathrm{N}}\right)$, which arise primarily from the source material contaminations and from the large background pressure of $\mathrm{O}_{2}, \mathrm{CO}_{2}, \mathrm{CO}, \mathrm{H}_{2} \mathrm{O}$, etc. during the sputtering process and possible nitrogen vacancies $\left(\mathrm{V}_{\mathrm{N}}\right)^{3,4}$ Previous reports of chemical vapor deposited $\mathrm{ScN}$ also contained halogen and other impurities arising from the reactants and other sources. ${ }^{31,32}$ Hole doped $p$-type ScN films have been developed by incorporating $\mathrm{Mg}_{\mathrm{x}} \mathrm{N}_{\mathrm{y}}$ inside $\mathrm{ScN}$ by magnetron sputtering. ${ }^{33}$ The $p$-type $\mathrm{ScN}: \mathrm{Mg}$ thin film also exhibited high Seebeck coefficients and large thermoelectric power factors at high temperatures. ${ }^{34}$ Synchrotron-based $\mathrm{x}$-ray absorption and photoemission measurements have demonstrated a rigid-band electronic structure of $\mathrm{ScN}$ across the $n$-type (the presence of unwanted $\mathrm{O}_{\mathrm{N}}$ ) to $p$-type (intentional $\mathrm{Mg}_{\mathrm{Sc}}$ hole doping) carrier transition regime, which results in the high Seebeck coefficient and thermoelectric power factor over the transition regime. ${ }^{35}$ In short, $\mathrm{ScN}$ has attracted great interest in recent years and could lead to several industrial applications in the near future.

While these interesting developments in $\mathrm{ScN}$ research over the last five-to-ten years are promising, most of the $\mathrm{ScN}$ thin films are deposited by low-vacuum magnetron sputtering, physical and chemical vapor deposition, vapor phase epitaxy methods, etc., where it is extremely challenging to control the impurity concentrations and achieve high crystalline quality. ${ }^{36,37}$ Moreover, for electronic, optoelectronic, and thermoelectric applications, it is important to achieve lower carrier concentrations and higher mobility simultaneously and reproducibly. For example, in ScN Schottky diodes, the carrier concentration needs to be reduced to less than $10^{18} \mathrm{~cm}^{-3}$ without altering its electron affinity, bandgap, and surface properties. Similarly, in $\mathrm{ScN}$ based metal/semiconductor superlattice heterostructures, lower carrier concentrations are essential for achieving thermionic energy conversion devices. In this regard, ultra-high vacuum molecular beam epitaxy (MBE) should be suitable for high-quality $\mathrm{ScN}$ growth and for controlling the impurity and dopant concentrations. MBE growth of high quality $\mathrm{ScN}$ and demonstration of interesting properties are also important for its integration with $\mathrm{GaN}$, InN, etc. for various applications. Unfortunately, only a few reports on the $\mathrm{ScN}$ depositions by MBE focus primarily on the growth and structural aspects ${ }^{38-43}$ with no reports on its thermoelectric properties. Given such a background and with a motivation to eventually develop ScN-based Schottky diodes and metal/semiconductor superlattice-based thermionic energy converters, in this Letter, growth and structural characterization of ScN epilayers deposited by plasma-assisted molecular beam epitaxy (PAMBE) in an ultrahigh vacuum environment are demonstrated alongside its impressive thermoelectric properties. In particular, highmobility and high-thermoelectric power factors in $\mathrm{ScN}$ films have been achieved and the physical mechanism of such transport properties is discussed, which will make it attractive for waste heat to electrical energy conversion applications.

$\mathrm{ScN}$ thin films with varying thicknesses are deposited by PAMBE inside an ultra-high vacuum chamber with a base pressure of $2 \times 10^{-10}$ Torr on the (001) MgO substrate at a growth temperature of $600{ }^{\circ} \mathrm{C}$. High purity Sc (99.95\% TREM) and $\mathrm{N}_{2}$ (99.99999\%) gas are used for the depositions, and a scandium K-cell temperature of $1320^{\circ} \mathrm{C}$ is used to achieve the desired Sc beam equivalent pressure (BMP) of $1 \times 10^{-7}$ Torr. The $\mathrm{N}_{2}$ flow rate was kept constant at 1.2 sccm with a forward rf-power of $375 \mathrm{~W}$. It is important to note that the Sc MBE source contained 500 ppm oxygen that is a known $n$-type dopant inside ScN. Except for the presence of oxygen, no other elements have a substantial presence in the source material. Further details about the growth process, deposition rate, etc. are presented in the supplementary material.

Post-growth structural characterization of ScN films using symmetric x-ray diffraction analysis show (002) oriented $\mathrm{ScN}$ crystal growth on (001) $\mathrm{MgO}$ substrates [see Fig. 1(a) for a representative spectrum of the $600 \mathrm{~nm}$ thick film]. The main 002 diffraction peak was located at $39.9^{\circ}$, which results in a ScN lattice constant of $4.51 \AA$, which is consistent with previous literature reports. ${ }^{38,42}$ The $\mathrm{MgO}$
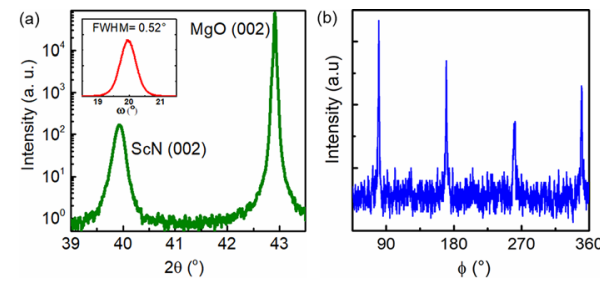

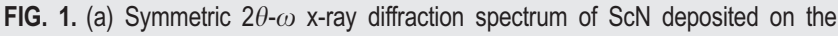
$\mathrm{MgO}$ substrate. ScN grows with 002 orientations on the (001) MgO substrate with a small FWHM of the rocking curve of $0.52^{\circ}$ (shown in the inset). (b) Asymmetric $\varphi$ scan of $\mathrm{ScN}$ that shows four equally spaced peaks representative of its epitaxial crystal growth. 
substrate 002 diffraction maxima were also found at $42.9^{\circ}$, representing its lattice constant of $4.21 \AA$, as expected. ${ }^{44}$ The full-width-at-thehalf-maxima (FWHM) of the rocking curve ( $\omega$-scan) corresponding to the main 002 peak was found to be $0.52^{\circ}$ in $\mathrm{ScN}$, which is indicative of epitaxial and textured film growth on $\mathrm{MgO}$ substrates. The FWHM of the rocking curve is consistent with the previous reports of $\mathrm{ScN}$ growth by radical-source $\mathrm{MBE}^{40}$ and is generally significantly smaller than that of the sputter-deposited films that exhibit much higher FWHM values between $\sim 1^{\circ}$ and $1.5^{\circ}$ depending on the growth conditions such as substrate temperature. ${ }^{14}$ Four equally spaced asymmetric ScN $111 \varphi$-peaks that are separated by $90^{\circ}$ apart from each other [see Fig. 1(b)] represents epitaxial ScN film growth on $\mathrm{MgO}$ substrates.

High-resolution (scanning)/transmission electron microscopy (HR(S)/TEM) analysis of the as-deposited ScN film reveals [see Fig. 2(a)] pyramidal grain growth with an epitaxial relationship of [001](001) ScN \| [001](001) MgO substrates within the grains. The pyramids are separated by inverted pyramid-like structures with grain boundaries that though maintains the out-of-plane epitaxy, but rotates the grain along the in-plane directions [see Fig. 1(a) in the supplementary material]. The inverted pyramid structures grow at a slightly faster rate and represent themselves as plateau-pyramid structures in the plan-view scanning electron microscopy (SEM) [see Fig. 3(a)] and atomic force microscopy (AFM) images [see Fig. 1(b) in the supplementary material]. It is important to note here that although no detailed $\mathrm{HR}(\mathrm{S}) / \mathrm{TEM}$ based microscopy analysis was performed in previous $\mathrm{MBE}$ growth of $\mathrm{ScN}$ on $\mathrm{MgO}$ substrates, previous AFM images had exhibited similar plateau-pyramid structures found here. ${ }^{38,42}$ The SEM images reveal flatter top surfaces with a root mean square (RMS) surface roughness of $1.13 \mathrm{~nm}$, measured using AFM. Along with the pyramidal grains, dislocation networks and stacking faults were also seen in both the pyramidal and inverted pyramid-like regions [see Fig. 2(a)]. (HR(S)/TEM) energy-dispersive spectroscopy (EDS) elemental maps clearly show that $\mathrm{Sc}$ and $\mathrm{N}$ atoms are uniformly distributed through the film, except along the grain boundary and dislocation lines that are dominated by oxygen atoms. Such a higher concentration of oxygen distribution along the defect lines most likely would have occurred due to the post-growth oxygen diffusion process.

Atomic-resolution HAADF-STEM images [see Fig. 3(b)] clearly show lattice fringes with cube-on-cube epitaxial growth as well as an atomically sharp and abrupt $\mathrm{ScN} / \mathrm{MgO}$ interface. Due to an $\sim 6 \%$ lattice-mismatch between the $\mathrm{ScN}$ and $\mathrm{MgO}$ substrates, misfitdislocations are observed in the STEM images. A detailed discussion on the origin and dynamics of extended defects such as grain boundaries and stacking faults including the effects of growth temperature is beyond the scope of the present study and will be presented later.

To gain an insight into the electronic properties of the $\mathrm{MBE}$ deposited $\mathrm{ScN}$ thin films, temperature-dependent Hall measurements are performed. At room temperature, the $\mathrm{ScN}$ film having a thickness of $450 \mathrm{~nm}$ exhibited the highest mobility of $127 \mathrm{~cm}^{2} / \mathrm{V} \mathrm{s}$, with an associated $n$-type (electron) concentration of $8.6 \times 10^{19} \mathrm{~cm}^{-3}$, which resulted in a room-temperature electrical conductivity of $1759 \mathrm{Scm}^{-1}$. Such a high mobility obtained in PAMBE-deposited $\mathrm{ScN}$ is consistent with the previous reports ${ }^{4,40,48-52}$ of $\mathrm{ScN}$ growth by radical source $\mathrm{MBE}$ and is higher than the mobility obtained with previous sputterdeposited ScN (see Table I in supplementary material). It is important to note here that the measured mobility is high despite the presence of extended defects such as grain boundaries and dislocation networks
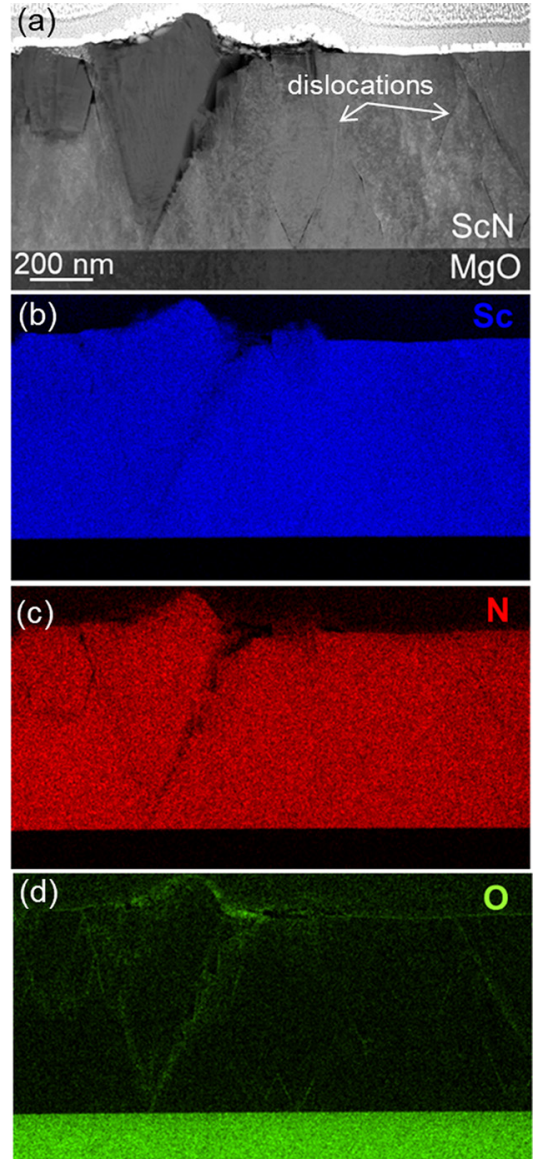

FIG. 2. (a) High-angle annular dark-field scanning transmission electron microscopy (HAADF-STEM) image of $\mathrm{ScN}$ deposited on the $\mathrm{MgO}$ substrate. An inverted pyramidal grain without the $[001]_{\mathrm{ScN}} \|[001]_{\mathrm{MgO}}$ orientation relationship is visible in the image. Dislocation lines are also visible in the image as highlighted. HRSTEMEDX maps show that Sc (b) and N (c) atoms are uniformly distributed over the entire $\mathrm{ScN}$ layer except for the grain boundaries. Oxygen (d) was found to cluster around the grain boundaries.

that are known to scatter electrons strongly. Subsequent partial or full removal of such defects should increase the mobility even further. The room temperature electrical conductivity of $1759 \mathrm{Scm}^{-1}$ of $\mathrm{ScN}$ is comparable to the electrical conductivity of well-known thermoelectric materials such as $\mathrm{Bi}_{2} \mathrm{Te}_{3}$ and $\mathrm{PbTe}$. $^{45-47}$

Low temperature $(50 \mathrm{~K}-400 \mathrm{~K})$ Hall measurements exhibited a decrease in the electron mobility from $172 \mathrm{~cm}^{2} / \mathrm{V} \mathrm{s}$ at $50 \mathrm{~K}$ to $106 \mathrm{~cm}^{2} /$ $\mathrm{V}$ s at $400 \mathrm{~K}$. To understand the details of the carrier scattering mechanism, the temperature-dependent mobility is modeled with a combination of ionized impurities $\left(\mu_{\text {ionized impurity }}\right)$ as well as dislocation scattering $\left(\mu_{\text {dislocation }}\right)$ of electrons as expressed in the following equation:

$$
\frac{1}{\mu_{\text {total }}}=\frac{1}{\mu_{\text {ionized impurity }}}+\frac{1}{\mu_{\text {dislocation }}} .
$$

The electron mobility increases at low temperatures due to ionized impurity scattering with a functional relationship, 


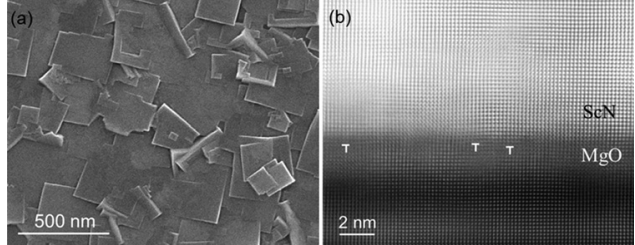

FIG. 3. (a) Plan-view SEM image of ScN showing inverted pyramidal grains growing at a slightly faster rate than the pyramidal grains. Within the grains, the surfaces are very smooth. (b) Atomically sharp and abrupt ScN/MgO interface along with misfit dislocations due to the lattice mismatch between $\mathrm{ScN}$ and $\mathrm{MgO}$.

$$
\mu_{\text {ionized impurity }}=A \mathrm{~T}^{1.5},
$$

where $T$ is the absolute temperature and $A$ is a constant. Dislocations can significantly scatter electrons in semiconductors in two distinct ways, especially when their densities are higher than $10^{8} \mathrm{~cm}^{-2} \cdot{ }^{53}$ First, a dislocation can be considered as a line charge and acts as a charged impurity center scattering electrons. Second, the strain field associated with dislocations can act as a scattering center similar to the deformation potential scattering. However, it is well known that the second effect is not appreciable for $n$-type semiconductors (such as $\mathrm{ScN}$ ) and influences primarily the $p$-type materials. Therefore, the dislocation scattering is primarily considered here as a line charge scattering center and expressed as

$$
\mu_{\text {dislocation }}=\left(\frac{3 q}{8 N_{d} R}\right) \frac{1}{\left(3 m^{*} k_{B} T\right)^{0.5}},
$$

where $N_{d}$ and $R$ are the dislocation density and dislocation core radius, respectively, $m^{*}$ is the electron effective mass, and $q$ and $\mathrm{k}_{\mathrm{B}}$ are the charge of an electron and the Boltzmann constant, respectively. An effective mass of $0.39 \mathrm{~m}_{\mathrm{o}}\left(\mathrm{m}_{\mathrm{t}}=0.28, \mathrm{~m}_{1}=2.08\right)^{33}$ was used for $\mathrm{ScN}$.

Figure 4(a) shows that the theoretical curve fits the experimental results very well. The fitting also revealed a dislocation core radius of $\sim 1 \mathrm{~nm}$ and a high dislocation density of $2 \times 10^{12} \mathrm{~cm}^{-2}$. Although the dislocation density of $\sim 10^{12} \mathrm{~cm}^{-2}$ is quite large, their numbers are quite consistent with the rough estimates from the microscopy analysis. In addition, since the grain boundaries can also act as line charge and scatter electrons, their number densities are also included in the $N_{d}$ calculation. Temperature-dependent Hall measurements also revealed that carrier densities of $\mathrm{ScN}$ exhibit a very small change in the temperature range of $50 \mathrm{~K}$ to $400 \mathrm{~K}$ [see Fig. 4(b)], suggesting that most dopants are ionized at the lowest temperature of $50 \mathrm{~K}$. Because of the decreasing mobility and relatively unchanged carrier concentration with temperature, the electrical conductivity [see Fig. 4(c)] of the ScN film shows a decrease with increasing temperatures representing its degenerate semiconducting nature.

Since $\mathrm{ScN}$ has shown potential for thermoelectric applications before, high-temperature thermoelectric properties such as the Seebeck coefficient and power factor (PF) are measured from $300 \mathrm{~K}$ to $950 \mathrm{~K}$. At $400 \mathrm{~K}$, the PAMBE-deposited ScN films exhibit a large Seebeck coefficient of $-107 \mu \mathrm{V} / \mathrm{K}$ that is slightly higher than the Seebeck coefficient of previous sputter-deposited ScN of $-95 \mu \mathrm{V} / \mathrm{K}$ at the same temperature. ${ }^{4}$ With the increase in temperature, the Seebeck coefficient increased monotonically and exhibited a maximum of $-175 \mu \mathrm{V} / \mathrm{K}$ at $950 \mathrm{~K}$. Concomitant with the increase in the Seebeck
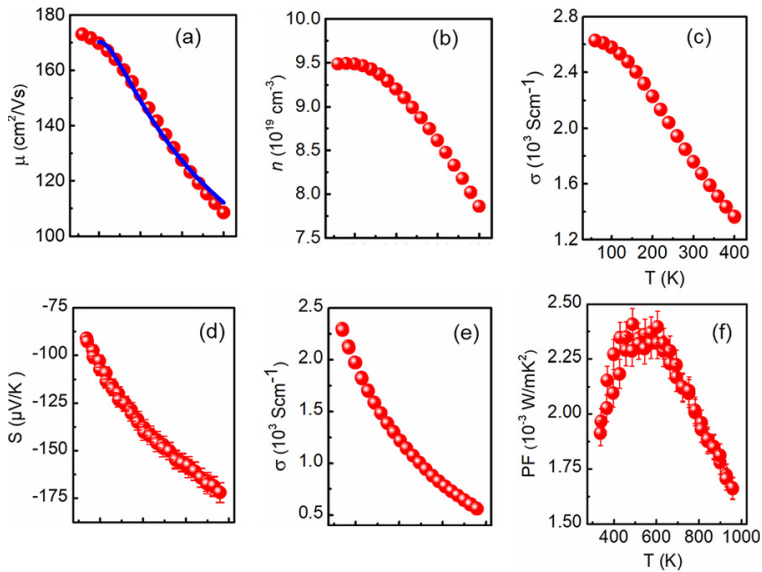

FIG. 4. Low temperature $(50 \mathrm{~K}-400 \mathrm{~K})$ (a) mobility, (b) carrier concentration, and (c) electrical conductivity of the $450 \mathrm{~nm} \mathrm{ScN}$ thin film. The mobility was found to decrease with increasing temperatures and is fitted with $\mathrm{Eq}$. (1) that accounts for a combination of ionized impurities and the dislocation scattering mechanism (blue line). High temperature ( $350 \mathrm{~K}-950 \mathrm{~K})$, (d) Seebeck coefficient, (e) electrical conductivity, and (f) thermoelectric power factor of the PAMBE deposited $300 \mathrm{~nm} \mathrm{ScN}$ thin film as a function of temperature. ScN films exhibit a maximum thermoelectric power factor of $2.3 \times 10^{-3}$ $\mathrm{W} \mathrm{m} \mathrm{m}^{-1} \mathrm{~K}^{-2}$ in the temperature range of $500-600 \mathrm{~K}$, which are higher than those of some well-known thermoelectric materials such as $\mathrm{Bi}_{2} \mathrm{Te}_{3}$.

coefficient, the electrical conductivity of the $\mathrm{ScN}$ films decreased from $2.2 \times 10^{3} \mathrm{~S} \mathrm{~cm}^{-1}$ at $300 \mathrm{~K}$ to $\sim 0.5 \times 10^{3} \mathrm{Scm}^{-1}$ at $950 \mathrm{~K}$. Previous Landauer and Boltzmann transport-based modeling analyses have demonstrated that due to its high carrier concentrations, the Fermi level of ScN is located $\sim 0.06-0.09 \mathrm{eV}$ above the conduction band minima, which leads to the degenerate semiconducting or metallic-like electrical conductivity behavior with increasing temperature. ${ }^{4}$

The thermoelectric power factor $\left(S^{2} \sigma\right)$ was found to increase initially in the temperature range of $300 \mathrm{~K}-600 \mathrm{~K}$, followed by a maximum, and then decreased monotonically [see Fig. 4(f)]. The highest power factor was achieved in the temperature range of $500 \mathrm{~K}-600 \mathrm{~K}$ with a value of $\sim 2.3 \times 10^{-3} \mathrm{~W} \mathrm{~m}^{-1} \mathrm{~K}^{-2}$, which is comparable with the power factors of previous sputter-deposited $\mathrm{ScN},{ }^{34}$ but higher than that of other well-known thermoelectric materials such as $\mathrm{La}_{3} \mathrm{Te}_{4}{ }^{54}$ and comparable with the values of high-temperature thermoelectric materials such as SiGe. ${ }^{55}$ It is important to note here that in spite of using the UHV-PAMBE method, the power factor of $\mathrm{ScN}$ remained comparable to that of sputter-deposited films because of the prsence of oxygen in the Sc source, which resulted in a high carrier concentration. Therefore, efforts should be made to use higher-purity Sc sources or to reduce the carrier concentration by hole doping without altering other electronic properties.

In spite of such a high power factor, the overall thermoelectric figure-of-merit (ZT) of ScN was found to be rather low (maximum ZT of $0.17 @ 500 \mathrm{~K})$ due to its high thermal conductivity measured using time domain thermoreflectance (see the supplementary material for details). The thermal conductivity of a $450 \mathrm{~nm} \mathrm{ScN}$ thin film decreased with increasing temperature due to Umklapp scattering [see Fig. 5(a)] and exhibited an exponent of -1.17 . It is important to note that the uncertainty in the thermal conductivity measurement at each temperature was larger than the normal due to the conductivity variations from area-to-area on the surface due to the changes in the surface 

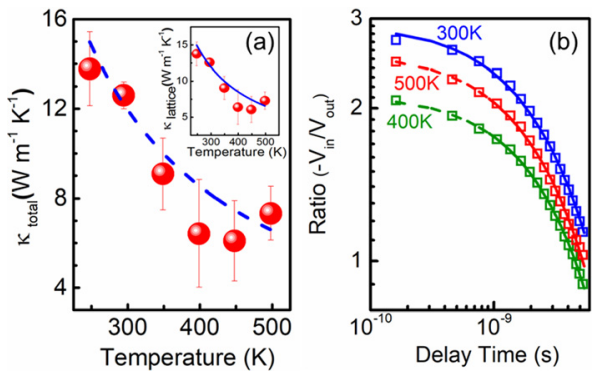

FIG. 5. (a) Total thermal conductivity of the $450 \mathrm{~nm} \mathrm{ScN}$ thin film measured from $250 \mathrm{~K}-500 \mathrm{~K}$ decreases with increasing temperature due to Umklapp scattering. The dashed line is drawn over the total thermal conductivity as a guide to the eye. (Umklapp model fitting of the lattice contribution to the total thermal conductivity is presented by the blue line in the inset). (b) Time domain thermoreflectance (TDTR) experimental data plotted as the ratio of the in-phase to out-of-phase lock-in voltages as a function of pump-probe delay time and the corresponding model fits used to determine the thermal conductivity of the ScN film. TDTR data are shown for three different measurement temperatures.

morphology resulting from the defects. Nevertheless, fitting of the TDTR data was quite well as exhibited in Fig. 5(b). Further research on alloying or nano-structure formation will be required to reduce the thermal conductivity of $\mathrm{ScN}$ without compromising the power factor to obtain higher ZT.

In conclusion, a high mobility of $127 \mathrm{~cm}^{2} / \mathrm{V} \mathrm{s}$ at room temperature and a large thermoelectric power factor of $\sim 2.3 \times 10^{-3} \mathrm{~W} \mathrm{~m}^{-1}$ $\mathrm{K}^{-2}$ in the temperature range of $500 \mathrm{~K}-600 \mathrm{~K}$ have been achieved in epitaxial ScN thin films deposited by plasma-assisted MBE. The epitaxial ScN films are found to grow with (002) orientations on the (001) $\mathrm{MgO}$ substrates with pyramidal grains separated by inverse pyramids and a sharp and abrupt $\mathrm{ScN} / \mathrm{MgO}$ interface. The low-temperature mobility was dominated by scattering from dislocations and ionized impurities, which revealed a high dislocation density consistent with HRTEM analysis. In spite of the presence of unwanted impurities such as oxygen, which resulted in a large carrier density, $\mathrm{ScN}$ films exhibit a high Seebeck coefficient and a high thermoelectric power factor. Although the thermoelectric power factor was found to be appreciably high, the overall ZT was limited to 0.17 due to the high thermal conductivity of $7.3 \mathrm{~W} / \mathrm{m}-\mathrm{K}$ at $500 \mathrm{~K}$. The present work will help attract attention toward $\mathrm{ScN}$ for its thermoelectric applications and engineer its structural and material properties for electronic and optoelectronic devices.

See the supplementary material for detailed information related to the growth and the characterization methods.

\section{AUTHORS' CONTRIBUTIONS}

D.R. and B.B. contributed equally to this work.

Data are available upon request from the authors.

D.R., B.B., and B.S. acknowledge the International Center for Materials Science (ICMS) and Sheik Saqr Laboratory (SSL) in JNCASR for support. B.S. acknowledges the Science and Engineering Research Board (SERB) of the Government of India, Start-Up Research Grant No. SRG/2019/000613 for financial support. M.S.M.G. wants to acknowledge financial support from No. MAT2017-86450C4-3-R and intramural CSIC Project No. 2D-MESES. M.G., V.B., and
A.I.K.P acknowledge the facilities of Sydney Microscopy and Microanalysis at the University of Sydney. Y.R.K. and P.E.H. appreciate support from a MURI program through the Office of Naval Research, Grant No. N00014-18-1-2429.

\section{REFERENCES}

${ }^{1}$ U. Guler, A. Boltasseva, and V. M. Shalaev, Science 344, 263 (2014).

${ }^{2}$ M. Sakhdari et al., Nano Energy 26, 371 (2016).

${ }^{3}$ S. Kerdsongpanya et al., Appl. Phys. Lett. 99, 232113 (2011).

${ }^{4}$ P. V. Burmistrova et al., J. Appl. Phys. 113, 153704 (2013).

${ }^{5}$ H. Uchiyama et al., Phys. Rev. Lett. 120, 235901 (2018).

${ }^{6}$ A. Shakouri, Annu. Rev. Mater. Res. 41, 399 (2011).

${ }^{7}$ J. M. Zide et al., Appl. Phys. Lett. 87, 112102 (2005).

${ }^{8}$ D. Gall et al., J. Appl. Phys. 84, 6034 (1998).

${ }^{9}$ H. A. Al-Atabi et al., Appl. Phys. Lett. 113, 122106 (2018).

${ }^{10}$ P. Eklund, S. Kerdsongpanya, and B. Alling, J. Mater. Chem. C 4, 3905 (2016).

${ }^{11}$ B. Saha, A. Shakouri, and T. D. Sands, Appl. Phys. Rev. 5, 021101 (2018).

${ }^{12}$ W. Feng et al., Physica B 405, 2599 (2010).

${ }^{13}$ N. Takeuchi, Phys. Rev. B 65, 045204 (2002).

${ }^{14}$ B. Saha et al., J. Appl. Phys. 114, 063519 (2013).

${ }^{15}$ A. Qteish et al., Phys. Rev. B 74, 245208 (2006).

${ }^{16}$ B. Saha et al., J. Appl. Phys. 107, 033715 (2010).

${ }^{17}$ V. Rawat et al., J. Appl. Phys. 105, 024909 (2009).

${ }^{18}$ M. Garbrecht et al., J. Mater. Sci. 51, 8250 (2016).

${ }^{19}$ M. Y. Shalaginov et al., Laser Photonics Rev. 9, 120 (2015).

${ }^{20}$ B. Saha et al., Phys. Status Solidi 252, 251 (2015).

${ }^{21}$ B. Saha et al., Phys. Rev. B 93, 045311 (2016).

${ }^{22}$ M. Saleemi et al., J. Mater. Chem. 22, 725 (2012).

${ }^{23}$ M. A. Moram et al., J. Appl. Phys. 106, 113533 (2009).

${ }^{24}$ M. E. Little and M. E. Kordesch, Appl. Phys. Lett. 78, 2891 (2001).

${ }^{25}$ S. Strite, J. Vac. Sci. Technol., B 10, 1237 (1992).

${ }^{26}$ C. F. Johnston et al., J. Cryst. Growth 311, 3295 (2009).

${ }^{27}$ M. A. Moram et al., J. Cryst. Growth 308, 302 (2007).

${ }^{28}$ M. Akiyama et al., Adv. Mater. 21, 593 (2009).

${ }^{29}$ S. Fichtner et al., J. Appl. Phys. 125, 114103 (2019).

${ }^{30}$ A. Zukauskaite et al., J. Appl. Phys. 111, 093527 (2012).

${ }^{31}$ J. P. Dismukes et al., J. Cryst. Growth 9, 295 (1971).

${ }^{32}$ J. P. Dismukes, W. M. Yim, and V. S. Ban, J. Cryst. Growth 13-14, 365 (1972).

${ }^{33}$ B. Saha et al., Appl. Phys. Lett. 110, 252104 (2017).

${ }^{34}$ B. Saha et al., Phys. Rev. B 97, 085301 (2018).

${ }^{35}$ S. Nayak et al., Phys. Rev. B 99, 161117 (2019).

${ }^{36}$ J. H. Edgar, T. Bohnen, and P. R. Hageman, J. Cryst. Growth 310, 1075 (2008).

${ }^{37}$ B. Biswas and B. Saha, Phys. Rev. Mater. 3, 020301 (2019).

${ }^{38}$ A. R. Smith et al., J. Appl. Phys. 90, 1809 (2001).

${ }^{39}$ J. L. Hall et al., J. Cryst. Growth 311, 2054 (2009).

${ }^{40}$ T. Ohgaki et al., J. Appl. Phys. 114, 093704 (2013).

${ }^{41}$ H. A. Al-Brithen, A. R. Smith, and D. Gall, Phys. Rev. B 70, 045303 (2004).

${ }^{42}$ H. Al-Brithen and A. R. Smith, Appl. Phys. Lett. 77, 2485 (2000).

${ }^{43}$ S. W. King, R. F. Davis, and R. J. Nemanich, J. Vac. Sci. Technol., A 32, 061504 (2014).

${ }^{44}$ R. W. G. Wyckoff, Crystal Structures, 1st ed. (Wiley, New York, 1963).

${ }^{45}$ K. T. Kim, K. J. Kim, and G. H. Ha, Electron. Mater. Lett. 6, 177 (2010).

${ }^{46}$ J. P. Heremans, C. M. Thrush, and D. T. Morelli, Phys. Rev. B 70, 115334 (2004).

${ }^{47}$ Z. H. Dughaish, Physica B 322, 205 (2002).

${ }^{48}$ J. Casamento et al., Appl. Phys. Lett. 115, 172101 (2019).

${ }^{49}$ Proceedings of the First Symposium on III-V Nitride Materials and Processes, edited by T. D. Moustakas, J. P. Dismukes, and S. J. Pearton (The Electrochemical Society Inc., Pennington, NJ, 1996), Vol. 96, p. 197.

${ }^{50}$ T. Ohgaki et al., J. Cryst. Growth 476, 12 (2017).

${ }^{51}$ J. S. Cetnar et al., Appl. Phys. Lett. 113, 192104 (2018).

${ }^{52}$ Y. Oshima, E. G. Villora, and K. Shimamura, J. Appl. Phys. 115, 153508 (2014).

${ }^{53}$ S. S. Li, Physics Semiconductor Electronics (Springer, New York, 2006).

${ }^{54}$ L. Gao et al., J. Phys. D. Appl. Phys. 45, 185303 (2012).

${ }^{55} \mathrm{H}$. Takiguchi et al., Mater. Trans. 51, 878 (2010). 